\title{
FHR Problems Solved by the Actocardiogram
}

\section{Kazuo Maeda*}

Department of Obstetrics and Gynecology (Emeritus), Tottori University Medical School, Yonago, Japan

\begin{abstract}
Aims: To clarify the problem related fetal heart rate.

Methods: Actocrdiogram was used to analyze the relation of fetal heart rate acceleration, physiological sinusoidal heart rate, long term variability and fetal movement burst, periodic fetal respiratory movements as well as minor fetal movement. The correlation of actocardiographicacceleration duration ratio to movement burst duration (A/B ratio) and fetal outcome was analyzed.

Results and conclusion: Fetal heart rate acceleration was evoked by fetal movement burst, moderate physiological sinusoidal heart rate was evoked by moderate fetal respiratory movement, and the long term variability was evoked by minor fetal movements. Since the loss of variability possibly caused by hypoxic fetal brain damage, Cesarean section is recommended to be performed prior to the loss of fetal heart rate variability. Fetal short term and long term outcome were predicted by the A/B ratio.
\end{abstract}

Keywords: Fetal heart rate, Fetal large, Medium and minor movements, Acceleration, Moderate and minor heart rate change, Fetal brain damage, Early C-section, Prevention of fetal brain damage, Prediction of fetal outcome, A/B ratio of actocardiogram

\section{Method}

\section{Actocardiogram}

Although fetal heart rate (FHR) was discussed in the relation to uterine contraction in the EMS or CTG, no fetal movement was studied in EFM or CTG, while the FHR was principally controlled by fetal movement, i.e. adult heart rate increases in any exercise to increases cardiac output by increasing the beating frequency. Also FHRincreased by fetal motion, which was recognized by fetal kicking by maternal perception, where the mother was afraid of fetal abnormality when fetal kicking reduced. However, fetal movement signal wasnot directly compared to fetal heart rate, because there was no precise method to directly record fetal body movement, i.e. maternal perception depends on maternal abdominal skin sensation, and a mechanical fetal motion detectorat maternal abdomen was indirect partial record of fetal movements [1]. The direct detection of fetal motion was intended by the author listening noises of the monitor sound of ultrasonic Doppler autocorrelation fetal heart rate monitor, which were listened at fetal motion observed at maternal abdomen. The author had studied fetal heart valve beating signals in the records ultrasonic Doppler fetal heart detector, and he noted the possibility to record fetal motion directly at fetal chest like as fetal heart beat detection, then he analyzed the Doppler frequency of fetal motion by a real-time FFT frequency analyzer to find fetal motion Doppler frequency to be 20 to $50 \mathrm{~Hz}$ when the ultrasound was $2 \mathrm{MHz}$. Already he studied that fetal heart beat Doppler frequency was higher than $100 \mathrm{~Hz}$ including the motion of cardiac valve, fetal heart wall and cardiac blood flow.

The facts promoted him to separate fetal motion Doppler signals from fetal heart beat Doppler signal using a band-pass filter with the -18 $\mathrm{dB} /$ Oct attenuation at 20 and $80 \mathrm{~Hz}$ frequency, where single ultrasound could simultaneously detect fatal movements and fetal heart beats using ultrasound Doppler technique. The author did it by himself with hand-made remodeling TN-400 ultrasonic Doppler autocorrelation fetal monitor cardiotocograph (TOITU, Tokyo) of which ultrasound frequency was $2 \mathrm{MHz}$, and a commercial $-18 \mathrm{~dB} / \mathrm{Oct} 20-80 \mathrm{~Hz}$ bandpass filter 1983. The results were successful, he studied basic technical character and clinical studies on fetal movement detection at fetal chest, and confirmed the linear relation of recorded signal amplitude and that of the movement, then reported the creation of new ultrasonic Doppler fetal movement record to the Journal of Japan Society of Obstetrics and Gynecology in 1984 [2]. World perinatal researchers tested the new machine and guaranteed its function to record every fetal movement [3-5]. The author asked TOITU (Tokyo) to produce commercial new ultrasonic Doppler fetal movement recorder (actocardiogram, named by the author)on 1984, and the first commercial model MT320 actoardiogram was provided, and followed by MT-325, MT-332, MT-333U, MT-430, MT-516, MT-517, MT-522 and MT-540 (TOITU, Tokyo). The price ofMT-516 is about 6,000 USD at present.

\section{Terminology}

Atocardiogram: 'Acto' is fetal movement and 'Cardio' fetal heart rate, namely 'Actocardiogram' means simultaneous record of fetal movement and heart rate. 'ACG' is abbreviation of actocaediogram. Uterine contraction is also simultaneously recorded in actual ACG machine to prepare the function of 'Cardiotocogram (CTG)'.

\section{FHR: Fetal Heart Rate.}

FHR acceleration: Triangular transient FHR increase. Duration is 15 or more secandamplitude 15 or more bpmafter 30 weeks, while 10 sec and $10 \mathrm{bpm}$ before 30 weeks.

The loss of acceleration is 'non-reactive FHR' before ACG.

Sinusoidal FHR: Two to 4 cycles of min sine wave-like smooth FHR waves of very ominous outcome. A physiological sinusoidal FHR resembles true sinusoidal FHR, while its outcome is benign without

${ }^{*}$ Corresponding author: Kazuo Maeda, Department of Obstetrics and Gynecology (Emeritus), Tottori University Medical School, Yonago, Japan, Tel: 81-859-22-6856; E-mail: maedak@mocha.ocn.ne.jp

Received July 04, 2014; Accepted September 17, 2014; Published September 20, 2014

Citation: Maeda K (2014) FHR Problems Solved by the Actocardiogram. J Health Med Informat 5: 168. doi:10.4172/2157-7420.1000168

Copyright: (c) 2014 Maeda K. This is an open-access article distributed under the terms of the Creative Commons Attribution License, which permits unrestricted use, distribution, and reproduction in any medium, provided the original author and source are credited. 
fetal obstacle. The true and physiological sinusoidal resembles each other, hard to distinguish by CTG, while physiological one is evoked by periodic fetal respiratory movements in ACG.

Deceleration: transient decreases of FHR. 'Nadir' is the lowest FHR in a deceleration. Periodic V-shaped decelerations are divided into early (ED) and late (LD) decelerations. The LD delays from uterine contraction and considered ominous sign. Variable U-shaped decelerations are divided into benign mild variable and ominous severe variable decelerations. Prolonged deceleration longer than $2 \mathrm{~min}$ is ominous.FHR baseline variability: The minor 5 to $24 \mathrm{bpm}$ variation of FHR baseline. The loss of variability is a very ominous sign.

Bradycardia: Decreased FHR baseline less than $110 \mathrm{bpm}$. An ominous hypoxic sign, particularly sudden and prolonged bradycardia. Two non-hypoxic fetal bradycaedia are fetal atrio-ventricular block and the sick sinus bradycardia.

Tachycardia: FHR baseline higher than $160 \mathrm{bpm}$. More than $200 \mathrm{bpm}$ fetal tachycardia is fetal cardiac pathology which needs pharmacologic treatment via placenta.

NRFS: non-reassuring fetal status, fetal asphyxia, fetal distress.

A/B ratio: Acceleration duration ratio to associated fetalmovement burst duration ratio. It was the sum of acceleration duration divided by the sum of movement burst duration in a observation period.

\section{Results}

\section{Developmental mechanism of FHR acceleration}

Triangular FHR accelerationsare accompanied, rather synchronized, with fetal movement bursts in fetal active state (Figure 1). Terao [6] found that the acceleration is produced in the mid-brain in the studies on anencephalic fetus.

Why the acceleration was triangular had not been clarified. Since the movement signal would stimulate the mid-brain to form the triangular acceleration, the correlation of acceleration and fetal movement should be studied, i.e. in the cross-correlation study of FHR and fetal movement signals, the correlation coefficient was the largest, when themovement signal wasdelayed for $7 \mathrm{sec}$ [7].

Since the $7 \mathrm{sec}$ will mean the processing time of movement burst, an electronic simulation test was studied, where $10 \mathrm{~Hz}$ wave groups (because fetal movement Doppler of $1 \mathrm{MHz}$ ultrasound is $10 \mathrm{~Hz}$ )were

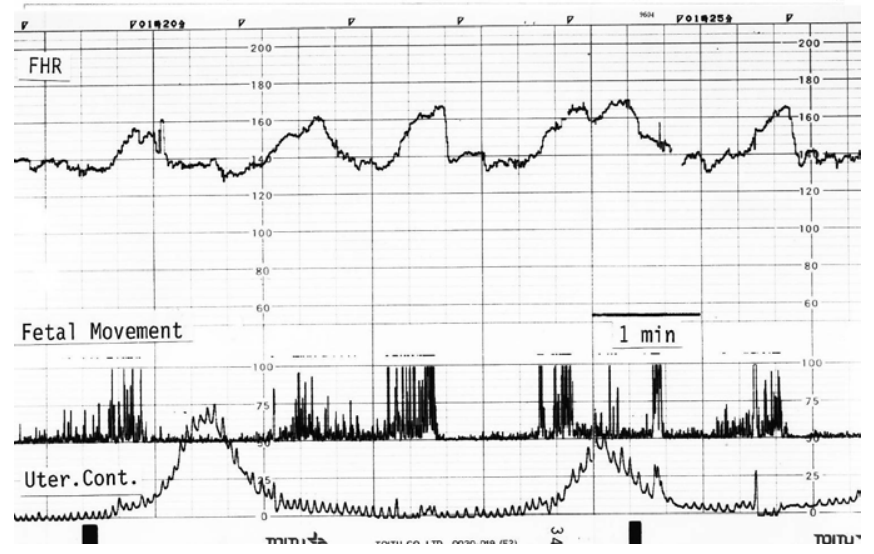

Figure 1: ACG recorded in active fetal state. Frequent triangular FHR accelerations are synchronized with fetal movement bursts.

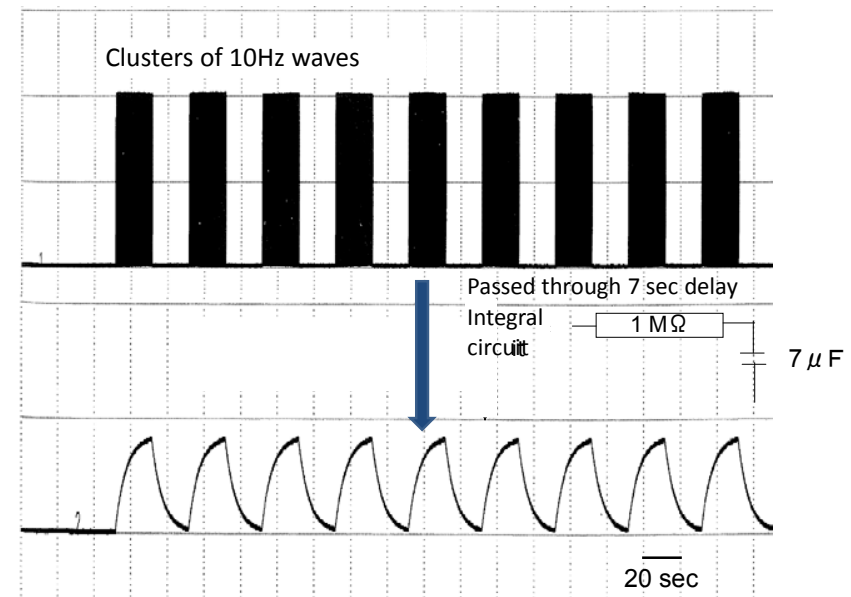

Figure 2: An electronic simulation test to form triangular wave from $10 \mathrm{~Hz}$ wave cluster. The $10 \mathrm{~Hz}$ wave group was passed through an integral circuit, of which time constant was $7 \mathrm{sec}$, i.e. the $10 \mathrm{~Hz}$ signals were delayed for $7 \mathrm{sec}$ in the integral circuit. The outputof the circuit was triangular curves similar to FHR acceleration. It will be concluded that the mid-brain center reacts fetal movement burst changing it to triangular acceleration.

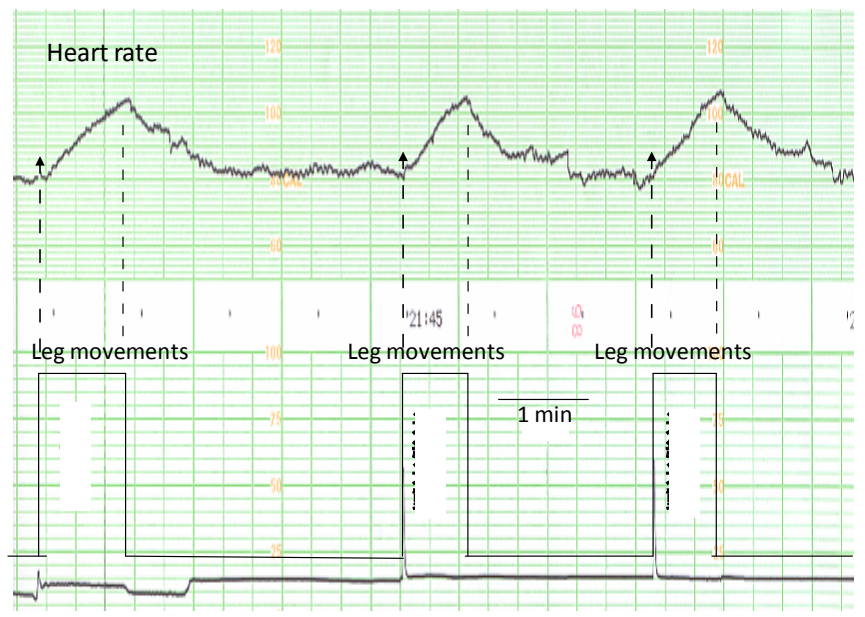

Figure 3: In a physiologic simulation test, triangular heart rate curve was obtained from repeated continuous leg motions. The result showed the reaction of mid-brain to change the continuous motion to triangular heart rate curve.

passed through an integral circuit, of which time constant was $7 \mathrm{sec}$ (the signal delay is $7 \mathrm{sec}$ ). Theresult obtained was not $10 \mathrm{~Hz}$ cluster, but triangular waves similar to FHR acceleration (Figure 2).

Another physiologic simulation test was the record of adult heart rate during continuous constant leg exercise. The heart rate curve was such triangular shape as FHR acceleration (Figure 3). Since the experimental person did not recognize the heart rate change, the transformation of movement to triangular heart rate would be conducted in the mid-brain but not in cortical brain. Therefore, the author confirmedthat fetal brain reacted the stimulation of fetal movement with $7 \mathrm{sec}$ delay.

\section{Physiologic sinusoidal FHR (Moderate baseline variation)}

Physiologic benign sinusoidal FHR was differentiated from true ominous sinusoidal one by the presence of synchronized periodic fetal 
respiratory movements [8]. Maeda traced the envelope of periodic fetal movements, and delayed it $7 \mathrm{sec}$, where the envelope coincided the physiologic sinusoidal fetal heart rate (Figure 4 ). The result clearly showed that fetal brain reacted fetal movements.

\section{Long term baseline variability}

Furthermore, the actogram of fetal resting state was augmented for 3 times, where minor movement signals appeared even in resting fetal state preserving FHR variability. It may suggest that minor movement stimulate the brain and minor FHR variability developed. Maeda further compared augmented FHR baseline and actogram, where minor fetal movement resembled the small variation of FHR (Figure 5).

\section{Discussion}

In summary, large FHR acceleration was evoked by large fetal movement burst, moderate size FHR variation was evoked by moderate

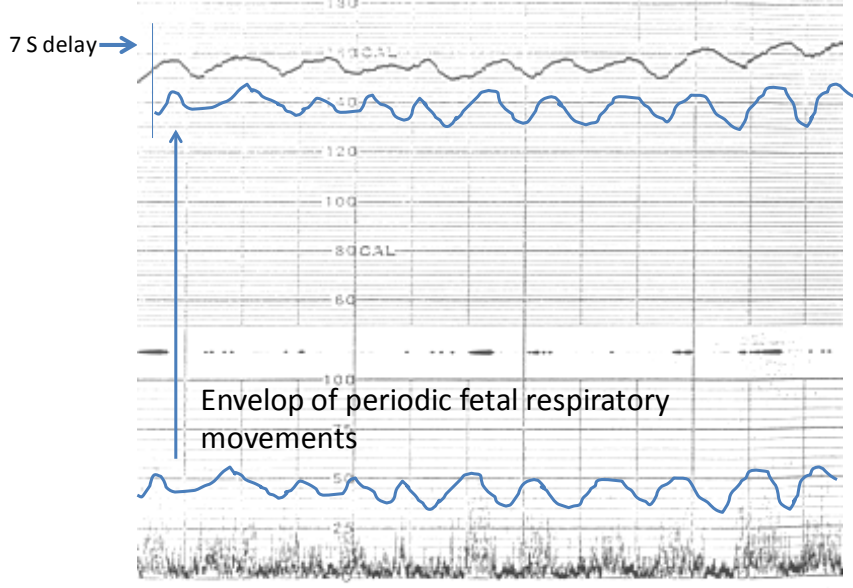

Figure 4: The envelope of periodic fetal respiratory movements was coincided physiologic sinusoidal FHR after the delay of envelope for $7 \mathrm{sec}$. The result shows the reaction of mid-brain to fetal movements to form moderate amplitude long term FHR variability.

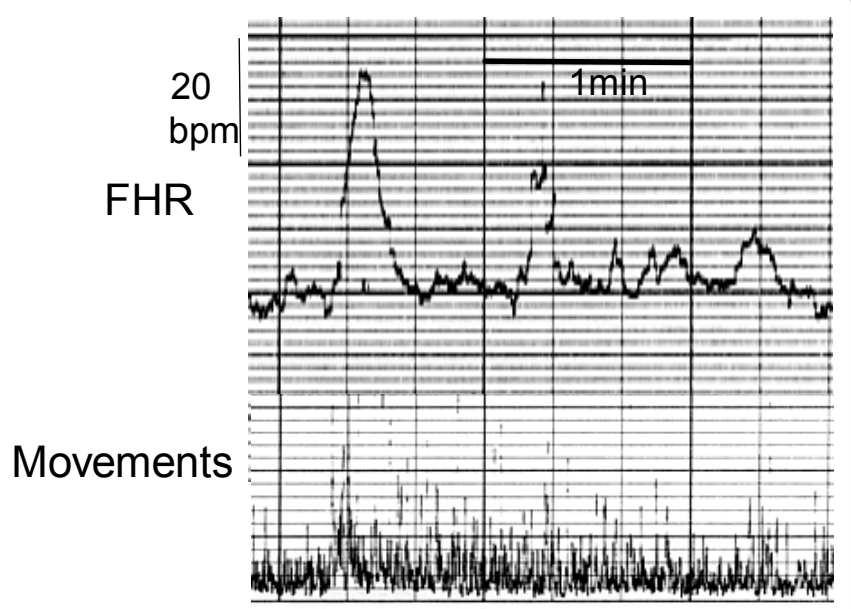

Figure 5: An augmented minor fetal movements provoked small long term FHR variability. Therefore, in summary, large fetal movement burst develop triangular FHR acceleration, moderate fetal movement develop moderate FHR variation, and minor fetal movement develop long term FHR variability by the reaction of fetal brain.

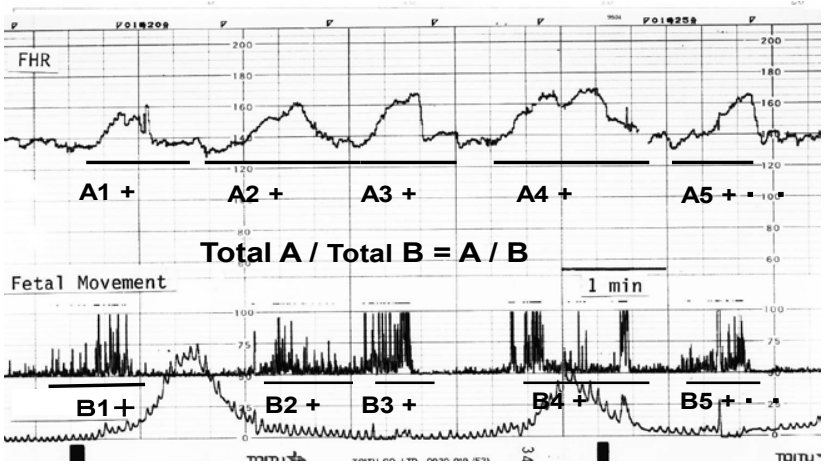

Figure 6: Calculation of $A / B$ ratio of $A C G$ : (Sum of $A) /($ sum of $B)$ is $A / B$ ratio.

fetal movements, and small FHR variability developed by the minor fetal movements. Alltransient FHR changes would be evoked by the reaction of fetal brain, probably in mid-brain.

As mentioned above, fetal movements evoke fetal heart rate increase by the reaction of fetal mid-brain. IUGR cases frequently suffer hypoxia, i.e.they frequently develop non-reactive FHR against fetal movement bursts, where FHR baseline variability is preserved. Some days or a week after the non-reactive FHR, severe NRFS appears with bradycardia or severe late deceleration and the loss of variability. Despite emergency C-section was performed, the outcome NTFS was more ominous including neonatal deaththan reactive FHR of positive acceleration without NRFS [9]. Since the loss of variability is partial phenomenon of fetal brain damage without reaction to fetal movements, there may be general brain damage possibly followed by neurological sequels or cerebral palsy. Therefore, initial loss of acceleration preserving variability will be early stage of hypoxia, and the loss of variability will be advanced severe hypoxia with fetal brain damage. Therefore, early C-section before the loss of variability would cure the fetus from severe brain damages (Figure 6).

\section{Clinical recommendation on the loss of FHR variability}

Since the loss of long term FHR variability (LTV) will possibly mean general brain damage due to hypoxia as discussed above, preventive C-section against neurological sequels is recommended to be performed before the loss of LTV, instead of the C-section after the loss of LTV, in general NRFS cases in fetal monitoring. Since the C-section is performed generally in severe NRFS including sudden continuous bradycardia, severe late or variable decelerations, prolonged decelerations, sinusoidal FHR, and so on, please confirm the presence of LTV before the C-section due to NRFS, or confirm that the hypoxic index (bradycardia duration $(\mathrm{min}) \times 100$ divided by the nadir FHR) is lower than 24 at the same time as positive LTV, because in severe NRFS, of which LTV may disappear, the hypoxia index was 25-26. The hypoxia index, however, should be further studied in cases of the loss of LTV.

\section{Complicated FHR changes in the hypoxia}

As discussed above, FHR shows complicated changes, i.e. the suppression or disappearance of acceleration but preserving FHR baseline variability in early stage of asphyxia, while the variability disappears in advanced hypoxia, according to the severe suppression or damage of fetal brain, while the suppression patternis complicated by the decrease of FHR in continuous bradycardia or deceleration, because 


\begin{tabular}{|c|c|c|c|c|c|}
\hline \multirow[t]{2}{*}{ Fetal disorder } & \multirow[t]{2}{*}{ Week of pregnancy } & \multirow[t]{2}{*}{$A / B$ ratio } & \multicolumn{2}{|c|}{ Apgar } & \multirow[t]{2}{*}{ Long term outcome } \\
\hline & & & $1 \mathrm{~min}$ & $5 \mathrm{~min}$ & \\
\hline NFRS(LOV) & 35 & 0 & 0 & 0 & 0 \\
\hline 18-Trisomy & 29 & 0.15 & 0 & 0 & 0 \\
\hline PIH, IUGR, LD & 35 & 0.34 & 2 & 9 & 8 \\
\hline Hydrops foetalis & 26 & 0.42 & 0 & 0 & 0 \\
\hline osteodysplasia & 29 & 0.5 & 1 & 4 & 1 \\
\hline Excencephaly, multiple anomalies & 37 & 0.54 & 1 & 3 & 3 \\
\hline Intestinal obstruction & 36 & 0.96 & 2 & 4 & 6 \\
\hline polydactyly & 37 & 1.01 & 8 & 9 & 9 \\
\hline Cardiac sick sinus bradycardia & 39 & 1.34 & 7 & 9 & 8 \\
\hline Endocardiac cushion defect & 37 & 1.2 & 9 & 9 & 8 \\
\hline megacystis & 38 & 1.22 & 8 & 9 & 8 \\
\hline Myotonic dystrophy & 32 & 1.26 & 9 & 9 & 6 \\
\hline Hydronephrosis & 39 & 1.31 & 9 & 9 & 9 \\
\hline Normal pregnancy & 37 & 1.43 & 8 & 9 & 10 \\
\hline hydrocephaly & 33 & 1.16 & 8 & 9 & 7 \\
\hline hydrocephaly & 30 & 0.73 & 3 & 4 & 4 \\
\hline Spina befida ventriculomegaly & 41 & 0.88 & 4 & 8 & 6 \\
\hline Spina bifida, corp call part .defect & 38 & 1.11 & 8 & 8 & 8 \\
\hline Interrup.ascend .aorta Type A & 40 & 1.34 & 9 & 9 & 8 \\
\hline Placental abruption & 38 & 1.32 & 9 & 9 & 9 \\
\hline
\end{tabular}

Table 1: Twenty Fetal disorders of which outcome was studied by A/B ratios of ACG.

Rabbits' heart rate (bpm)

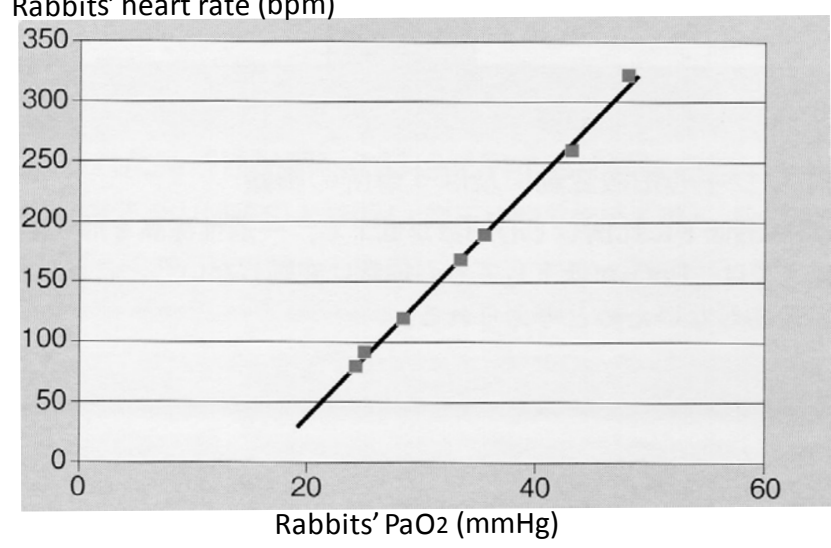

Figure 7: $(A)$ Correlation of $A / B$ ratio $(X)$ and 1 min Apgar score $(Y)$ in 20 fetal disorders (Table 1): $Y=7.68 X-1.75, R^{2}=0.85, p=0.001$, (B) Correlation of $A / B$ ratio $(X)$ and 5 min Agar score $(Y)$ : $Y=6.44 X+0.58, R^{2}=0.68, p<0.001$.

of the excitation of parasympathetic center of the medulla oblongata, which will be a protective reaction of autonomic nerve system activated by low $\mathrm{PaO}_{2}$ [10], i.e. rabbit heart rate decreases parallel to lowering of $\mathrm{PaO}_{2}$ (Figure 7). In the most severe hypoxic fetal brain damage, all brain functions will belost,therefore FHR baseline is flat and constant due to the automaty of fetal heart, which may be calledfetal brain death.

\section{Fetal outcome predicted by the $A / B$ ratio of actocardiogram}

The acceleration duration ratio to movement burst duration (A/B ratio) was parallel to the severity of fetal central nervous system lesions $[11,12]$, furthermore in common fetal disorders the A/B ratio closely correlated Apgar score and long-term outcome of the infants [13].

One and 5 min Apgar scores were closely correlated to A/B ratios of ACG recorded in prenatal stages (Figure 8). The long term outcome after birth was voluntarily determined into numeric values, which

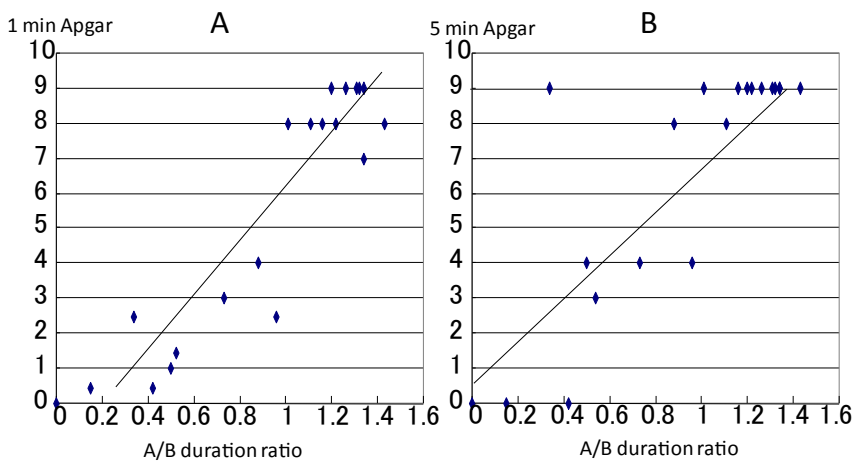

Figure 8: $(A)$ Correlation of $A / B$ ratio $(X)$ and long term numerical outcome $(Y)$. (B) Numerical long term outcome. $Y=6.42 X+0.05, R^{2}=0.71, p<0.001$.

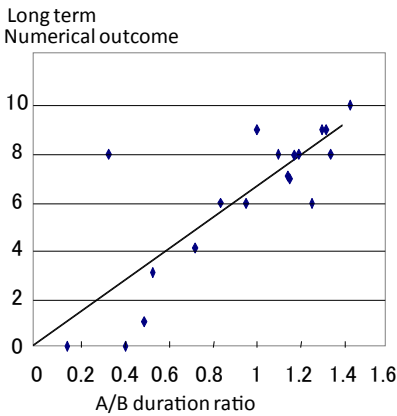

\begin{tabular}{lc}
\hline Long term status & $\begin{array}{c}\text { Numerical } \\
\text { outcome }\end{array}$ \\
\hline Intrauterine death & 0 \\
Death in day 1 & 1 \\
Death within 1 week & 2 \\
Death within 1 year & 3 \\
Death within 5 years & 4 \\
Spastic quadriplegia & 5 \\
Growth retardation & 6 \\
Mental retardation & 7 \\
Well and change hospital & 8 \\
Well with mild sickness & 9 \\
Healthy & 10 \\
\hline
\end{tabular}

Figure 9: Close correlation of rabbit heart rate and $\mathrm{PaO}_{2}$ below $50 \mathrm{mmHg}$.

was closely correlated fetal A/B ratio of ACG (Figure 9). These facts indicate that the A/B ratio of ACG, which was the FHR acceleration durationstandardized by fetal movement, is capable to predict short and long term fetal outcomes, i.e. the $\mathrm{A} / \mathrm{B}$ ratio would be valuable to 
be an important parameter in the fetalB monitoring. Although 'nonreactive FHR' was already reported to be an ominous sign of FHR, more detailed quantitative acceleration duration data,standardized by fetal movement, generally indicate fetal outcomes.

\section{References}

1. Maeda K, Kimura S, Nakano H, Pathophysiology of Fetus (1969) Fukuoka Printing, Fukuoka, Proc XXI Annual Conv Jap Obstet and Gynecol Society, Kanazawa, 1969.

2. Maeda K (1984) New ultrasonic Doppler fetal actograph and continuous recording of fetal movement. Nihon Sanka Fujinka Gakkai Zasshi 36: 280-288.

3. DiPietro JA, Costigan KA, Pressman EK (1999) Fetal movement detection: comparison of the Toitu actograph with ultrasound from 20 weeks gestation. $J$ Matern Fetal Med 8: 237-242.

4. Schwöbel E, Fallenstein F, Huch R, Huch A, Rooth G (1987) Combined electronic fetal heart rate and fetal movement monitor--a preliminary report. $J$ Perinat Med 15: 179-184

5. Wheeler T, Roberts K, Peters J, Murrills A (1987) Detection of fetal movement using Doppler ultrasound. Obstet Gynecol 70: 251-254.

6. Terao T, Kawashima Y, Noto H, Inamoto $Y$, Lin TY, et al. (1984) Neurological control of fetal heart rate in 20 cases of anencephalic fetuses. Am J Obstet Gynecol 149: 201-208.
7. Terao T, Kawashima Y, Noto H, Inamoto Y, Lin TY, et al. (1984) Neurological control of fetal heart rate in 20 cases of anencephalic fetuses. Am J Obstet Gynecol 149: 201-208.

8. Takahashi H (1990) Studies on cross correlation coefficient of fetal heart rate and fetal movement signals detected by ultrasonic Doppler fetal actocardiogram. Acta Obstet Gynecol Jpn 42: 443-449.

9. Ito T, Maeda K, Takahashi H, Nagata N, Nakajima K, et al. (1994) Differentiation between physiologic and pathologic sinusoidal FHR pattern by fetal actocardiogram. J Perinat Med 22: 39-43.

10. Teshima N (1993) Non-reactive pattern diagnosed by ultrasonic Doppler fetal actocardiogram and outcome of the fetuses with non-reactive pattern. Nihon Sanka Fujinka Gakkai Zasshi 45: 423-430.

11. Maeda K, Morokuma S, Yoshida S, Ito T, Pooh RK, et al. (2006) Fetal behavior analyzed by ultrasonic actocardiogram in cases with central nervous system lesions. J Perinat Med 34: 398-403.

12. Morokuma S, Fukushima K, Yumoto Y, Uchimura M, Fujiwara A, et al. (2007) Simplified ultrasound screening for fetal brain function based on behavioral pattern. Early Hum Dev 83: 177-181.

13. Maeda K, Iwabe T, Yoshida S, Ito T, Minagawa Y, et al. (2009) Detailed multigrade evaluation of fetal disorders with the quantified actocardiogram. $J$ Perinat Med 37: 392-396.

14. Umezawa $\mathrm{J}$ (1976) Studies on the relation between heart rate and $\mathrm{PaO}_{2}$ in hypoxic rabbit: a comparative study for fetal heart rate change during labor. Acta Obstet Gynecol Jpn 28: 1203-1212. 